\title{
ENTRETIEN AVEC GERTY DAMBURY ${ }^{1}$
}

\begin{abstract}
Les Cahiers de la LCD : Chère Gerty Dambury, merci d'avoir accepté cet entretien. On vous connaît pour votre engagement artistique au théâtre, mais aussi en tant qu'écrivaine de romans, de poèmes, sur des thématiques au cœur des préoccupations de notre revue dont la lutte contre les discriminations. Qu'est-ce qui vous pousse, dans vos écrits, dans vos prises de positions citoyennes, en tant que personne, à vous engager dans cette lutte?
\end{abstract}

Les discriminations qui motivent mon engagement sont de plusieurs ordres : elles concernent les personnes étrangères en situation de migration, les personnes victimes de discriminations en fonction de leur origine et de leur couleur de peau et les femmes. En réalité, je me retrouve dans ces trois catégories. Je m'y suis retrouvée très jeune, étant une personne de sexe féminin, noire, ayant émigré dès l'âge de 14 ans. Le fait de m'opposer aux discriminations n'a donc pas été, pour moi, dans un premier temps, lié à des considérations générales de type philosophique ou œcuménique, mais il s'agissait de réactions à des situations vécues que je vois encore à l'œuvre, envers moi-même et envers d'autres, des situations que je reconnais, même lorsqu'elles ne me concernent pas directement.

L'élargissement de mon combat, d'un point de vue personnel à un point de vue général s'est fait par un cheminement d'ordre politique, au sens le plus large du terme, c'est-à-dire par le biais d'une prise de conscience de l'organisation du mode de fonctionnement des sociétés dans lesquelles j'étais amenée à vivre. Le sentiment de vivre dans une société inégalitaire, la Guadeloupe, dans laquelle se perpétuait une organisation hiérarchique injuste, basée sur l'origine sociale et ethnique, vestige de l'esclavage, je l'ai éprouvé dès que j'ai observé comment se comportaient les gens autour de moi. Les Guadeloupéens noirs et indiens se débattaient pour gravir les échelons, se fixaient des objectifs pour lesquels ils devaient déployer trois fois plus d'efforts que les autres, ce qui, en eux, occasionnait de la frustration, de la colère et quelquefois un immense sentiment d'impuissance. À l'inverse les personnes blanches dotées du pouvoir économique et institutionnel jouissaient d'une assurance qui m'interrogeait. Et puis, ceux dont on parle très rarement : les personnes blanches très pauvres. Pour ceux-là, quelle complication cela devait être que de se voir renvoyé à leur isolement, à un vague reste de sentiment de supériorité, violemment contredit par la réalité de leur condition. Ils avaient à faire face à cette contradiction en eux : ne pas vouloir se mélanger aux Noirs et aux Indiens et tout à la fois, être totalement exclu des lieux où les Blancs détenteurs du pouvoir se tenaient, sur leurs mornes, en dominants qui, quelquefois, éprouvaient le besoin de «faire société ».

Il se trouve que, régulièrement, et à toutes les occasions, la référence à cette organisation inégalitaire revient au-devant de la scène, dans ces pays, départements et territoires « outremer» : ces questions ne sont pas réglées. Je ressens ces formes de discriminations sur le territoire hexagonal : dans l'organisation de l'espace, dans les ruptures entre le centre et la périphérie, dans la perpétuation d'un modèle hiérarchique issu d'une histoire coloniale que

\footnotetext{
${ }^{1}$ Cet article est paru dans le premier HS des Cahiers de la LCD (« Droits culturels et lutte contre la discrimination », 2018) pp : 74-82
} 
beaucoup continuent à vouloir oublier sans en extirper les racines. Elles sont à l'œuvre à l'école, au travail, dans la vie de tous les jours. Elles s'expriment par les préjugés, les idées toutes faites sur les Asiatiques, les Maghrébin.e.s, les Africain.e.s, les Antillais.e.s. Elles s'expriment dans les attributions de rôles et de postes, dans les rejets, les regards, les mots entendus, les attitudes de méfiance qui se trouvent renforcées par des campagnes de diabolisation des réfugié.e.s, des immigré.e.s, des femmes voilées, des jeunes des « quartiers ». Car enfin, tandis que l'on déplace le curseur vers davantage de « rencontre », de «vivre-ensemble», de «mixité sociale», on s'invente de nouveaux boucs émissaires. En sorte qu'il existe toujours des personnes qui sont exclues, discriminées: Roms, femmes noires, femmes voilées. Toutes ces personnes portent en elles une part de mon histoire et de ce fait, je me sens concernée par ce qu'elles vivent.

\section{D'après vous, entre le roman, les poèmes, le théâtre, y a-t-il des supports plus aisés que d'autres pour faire passer votre message?}

Je ne crois pas aux supports plus aisés. Je crois plutôt à la force des relais, aux opportunités offertes aux créations pour rencontrer les différents publics auxquels ces œuvres s'adressent. J'écris de la poésie, du théâtre, des romans et des textes de réflexion, courts et longs. Il me semble qu'à un moment donné, chacun de ces modes d'expression m'a permis de faire entendre l'une de mes préoccupations. Je ne parlerais pas de «message » à proprement parler. La plupart du temps, je ne pars pas de l'idée de message à transmettre. J'ai plutôt le désir de me laisser réagir à une situation et je laisse la place au mode d'expression qui me vient à ce moment-là. Je prendrai pour exemple la suite poétique Qui vive? que j'ai écrite durant le mouvement du LKP (Lyannaj Kont Pwofitasyon) en Guadeloupe. Ce mouvement ciblait les inégalités en combinant des luttes contre plusieurs espaces de discriminations : envers les handicapés, les personnes à faible revenu, les locataires vivant dans des habitations à loyer modéré subissant des menaces répétées d'expulsion, etc. Jour après jour, j'ai ressenti la présence ou l'absence de personnes. Je ne me suis pas focalisée sur les messages, mais sur les personnes et la manière dont elles vivaient ce mouvement. Que disaient ces corps ? Celui d'une amie malade, fiévreuse - décédée depuis - et cependant présente dans les rues, dans les manifestations, comme si ce mouvement pouvait constituer un espace de guérison. Que me racontait l'absence d'une autre, ses tergiversations et sa brusque apparition au sein du cortège de manifestants ? Que pouvait signifier la présence silencieuse et quasi invisible de personnes âgées qui, instinctivement, offraient ce qu'elles pouvaient à ce moment de révolte ? Quels chemins empruntent les uns et les autres pour l'expression de ces choses contenues et qui soudain explosent? La poésie pouvait me permettre de dire cela, par petits tableaux impressionnistes, du moins, je crois. Ces poèmes vivent leur vie. Il me revient, ici ou là, que tel texte a été lu, partagé, reproduit. Dans ce recueil, le poème en hommage à Jacques Bino, le syndicaliste tué durant le mouvement LKP a été republié par les éditions Bruno Doucey, dans deux ouvrages collectifs différents. Pour moi, ce signe est fort. Dans le même temps, je ne suis pas le ou la poète officielle de tel ou tel groupe, de telle ou telle théorie, je n'invente pas de théorie. J'analyse un peu pour m'éclairer moi-même, mais j'ai une sainte horreur des écrivain.e.s ou auteur.e.s délivrant une parole sanctifiée. 
Vous avez participé à la création du collectif $\mathbf{R}=$ Respect : quelle en est l'origine et surtout quels en sont les objectifs?

Le collectif $\mathrm{R}=$ Respect a été créé en réaction aux insultes proférées à l'encontre de Christiane Taubira en 2013. Un collectif de femmes noires s'est constitué, a rédigé une lettre ouverte au président de la République, François Hollande. Cette lettre a récolté environ 6000 signatures et a largement participé à la mobilisation contre le racisme. Nous avons reçu une réponse florentine de la directrice de cabinet de François Hollande mais nous avons observé que les mobilisations autour des «people » étaient bien plus intéressantes et médiatisées par certains et certaines que les actions d'un collectif constitué de militantes féministes noires. Le militantisme n'a de valeur aux yeux du plus grand nombre que s'il est porté par des figures cinématographiques, des chanteuses et des chanteurs. Je ne dis pas cela par amertume mais parce que cela constitue un changement majeur dans notre société. Ce constat est valable dans bien des cas. L'on a fait plus de cas du décès de Mireille Darc - contre qui ou à propos qui je n'ai pas grand-chose à dire, elle ne constitue qu'un exemple - que du décès de Jean-Pierre Kahane, très grand mathématicien, fondateur de l'université Paris-Orsay et qui a beaucoup milité et œuvré sur la question des apports des sciences au bien-être des humains.

Le collectif $\mathrm{R}=$ Respect a échoué à faire entendre sa voix de façon durable sur les questions du racisme, des discriminations et des inégalités. Il n'a pas duré. Cependant, toutes les personnes à l'origine de la création de ce collectif sont investies ailleurs: dans l'accompagnement des réfugiés syriens, dans la défense des femmes lesbiennes de couleur qui subissent des violences, en Afrique du Sud, en Inde et ailleurs, du fait de leurs orientations sexuelles (les LOC's - Lesbians Of Color) dans la diffusion des écrits et réalisations des Noir.es (Blakistoua), dans la lutte contre les discriminations dans le milieu du spectacle (Décoloniser les Arts), etc. Les luttes contre le racisme se déploient de cette façon également.

La thématique coloniale, très présente dans votre travail, passe aussi par votre engagement dans le collectif Décoloniser les arts. Si décoloniser les arts, c'est promouvoir la diversité, d'après vous, comment décoloniser les représentations ? La visibilité suffit-elle ? Cela doit-il passer par la question mémorielle ?

Trois termes m'interrogent dans votre question : celui de «diversité », celui de «visibilité » et l'expression «question mémorielle ». Je saisis l'occasion pour dire que l'un des axes de bataille de l'association Décoloniser les Arts, c'est précisément la question du langage et du vocabulaire. En France, on tourne en rond autour de questions qu'on ne sait pas aborder et on cherche des expressions pour ne pas dire ce que l'on devrait dire.

Parlons de la «diversité ». La question, que nous posions il y a deux ans, commence à rencontrer un certain écho. Nous retournions le problème en mettant en évidence le fait que parler de «diversité » signifiait qu'il y avait une norme à partir de laquelle on « divergeait » d'une certaine façon. Ceux qui étaient renvoyés à leur «diversité » étaient, en quelque sorte, en position d'écart par rapport à la norme basique. L'homme blanc. Les mouvements luttant pour l'égalité entre les hommes et les femmes ont mis en avant cette expression de "l'homme blanc de 50 ans ». Celui-là est comme naturellement appelé à occuper les fonctions, toutes les fonctions les plus élevées dans la hiérarchie sociale puisqu'il est la norme. À partir de là, les revendications posées par les femmes, les personnes visiblement non-blanches, les personnes handicapées, ont fait désigner ces formes humaines - je suis volontairement provocatrice - 
sous le vocable de «divers ». J'aime cette idée que, d'une certaine façon, nous avons forme humaine, mais nous constituons l'écart dans l'humain. C'est violent de le recevoir de cette façon, mais c'est ce que dit le terme « diversité » et nous le recevons avec la même violence. Donc, peut-être serait-il temps de se débarrasser définitivement de cette appellation qui ne constitue qu'une litote permettant de masquer son embarras à nommer celui ou celle que l'on exclut de façon totalement volontaire, en se basant sur des manières dépassées de considérer l'organisation de la société, hormis quelques exceptions qui viennent confirmer la règle.

Le terme «visibilité » est un piège extraordinaire ! Je me rappelle avoir eu un conflit avec un supérieur lorsque j'enseignais encore. Je m'étais fait insulter en tant que femme, avec des propos d'une rare vulgarité par des collègues enseignants. Au courrier que j'ai fait parvenir à mon chef d'établissement, par lequel je lui demandais d'intervenir auprès de ces collègues, il m'a été répondu, par ce supérieur : " y en a marre des minorités visibles ».J'étais d'abord, à ses yeux, une minorité visible. Un individu de couleur noire. Quelles étaient toutes les autres facettes de ma personnalité ? Qui étais-je véritablement? Les insultes que j'avais reçues me visaient clairement en tant que femme, mais il a préféré mettre en avant ma couleur de peau.

Très bien. Je suis donc piégée par des définitions parcellaires de mon identité. Je ne peux qu'être partiellement reconnue dans cette organisation de la pensée qui n'est pas en mesure de prendre en compte l'entièreté de ma personne.

Il me semble que c'est là un mode de pensée qui est à l'origine de la plupart des conflits, des préjugés, des violences à l'encontre de ceux et celles que nous croisons. Qu'est-ce que je choisis de voir chez celui ou celle qui passe, qui entre et qui demeure ?Puis-je subodorer tout ce qu'il ou elle est en mesure de m'apporter, de m'enseigner ou vais-je de façon systématique le ou la découper en petites tranches, de couleur, de genre, d'accent, de langue ?Partant de là, à partir de quel critère vais-je décider que je vais embaucher ou nommer un ou une noire, une personne apparemment d'origine maghrébine, une personne d'apparence asiatique? Évidemment, l'on parlera des qualités de cette «visible», mais en réalité, sa «visibilité » serait la principale raison de sa présence en un lieu donné. Il faut aller plus loin que cela. Il faut parvenir à se défaire de ses manières de regarder autour de soi. Il faut se déprendre du formatage de son monde intérieur. C'est certainement le plus difficile.

À tout cela, vous ajoutez la «question mémorielle ». Nous voilà au cœur des polémiques les plus violentes de ces dernières années.Pourquoi fait-on de certains pans de la mémoire une «question », c'est-à-dire un problème quand, par ailleurs, on ne se prive pas de faire de Charles Martel celui qui arrêta les Arabes à Poitiers ? Ou encore, quand on ne se prive pas de faire de Christophe Colomb celui qui a «découvert l'Amérique»? Ou encore, dernier exemple, quand on ne se prive pas de faire de Victor Schœlcher l'homme providentiel quasi unique de l'abolition de l'esclavage ? Je parle, en particulier en pensant à tous ceux qui dénoncent la «question mémorielle ».C'est donc bien qu'il y a choix de mémoire et refus d'autres mémoires. Or, si l'on n'est pas totalement aveugle à la réalité de l'histoire du monde, on devrait savoir que les voix finissent toujours par se faire entendre et que les oublié.e.s de l'Histoire se hisseront d'une manière ou d'une autre et quelquefois de la manière la plus violente qui soit. C'est quasiment un principe physique. Un corps que l'on tente de noyer finit toujours par remonter.

Je suis désolée d'avoir à faire un si grand détour pour parvenir à une réponse à votre question principale, mais elle était truffée de pièges... Au final, je dirais que « décoloniser les arts », ce 
n'est pas promouvoir la « diversité ». C'est un point de vue certainement très personnel. Je ne plaide pas pour mettre des «visibles » ici et là.Décoloniser les arts, c'est véritablement aller au cœur de questions qui sont posées, non pas aux dits "divers », mais à ceux qui se voient comme une majorité naturelle. Décoloniser les arts, c'est sortir de son entre-soi. Décoloniser les arts, c'est accepter de se dire qu'on n'a pas « l'art infus », décoloniser les arts, c'est mieux se connaître pour mieux se dire soi-même, ce soi-même étant composé de nous tous, quels que soient nos genres, nos couleurs, nos entièretés ou nos parcelles.

Décoloniser les arts, c'est se poser la question du socle sur lequel s'appuie notre perception de ce que sont l'art et l'artiste. Quand et à quel moment, et pour quelles raisons, pour occuper quelle place et quel rôle, la notion d'art, dans sa sacralité, est-elle intervenue ? Cet artiste sacré peut-il être une femme ? Bien sûr, il en existe, mais on joue sur l'exceptionnalité de la chose ! Peut-il être un Noir ? Bien sûr, il en existe mais on joue sur sa proximité et sa ressemblance avec la «norme ». On se comprend... Et si l'art c'était de ne pas tout comprendre, tout saisir parce que ça parle à ce que vous connaissez déjà et de devoir s'interroger ? Peut-il être un «divers handicapé » ? C'est à cet endroit que je pose la question de la décolonisation des arts. Pas tout à fait dans le nombre de «visibles » qui seraient chargés de porter de surcroît le poids de la «question mémorielle », qu'on leur laisserait tout en se disant : «Mon dieu, encore un truc identitaire !»

\section{Selon votre expérience, dans le monde de la culture, comment à la fois lutter contre les inégalités et privilégier la diversité, sans tomber dans le tokenisme ?}

Je pense qu'en général, et pas seulement dans le monde de la culture, pour lutter contre les inégalités, il est essentiel d'offrir les mêmes chances à tous et à toutes. Évidemment, c'est bateau, mais ça n'existe tellement pas ! Il y a tant d'obstacles invisibles à l'obtention de ce qui, normalement vous est dû, de ce qui est en principe à votre disposition !

Hier encore, j'observais un père qui me paraissait être Africain - mais qu'est-ce que je savais d'autre que le fait qu'il était noir - qui poussait sa dernière-née dans sa poussette tandis que l'aînée voulait pénétrer dans la salle jeunesse de la bibliothèque dans laquelle j'étais allée emprunter un ouvrage. Ils étaient tous les trois à la porte de cette salle jeunesse. Hésitants. Intimidés. La petite avait un air bravache. Le père avait un sourire gêné et moi, depuis la cage de l'ascenseur, je pensais, le plus fort possible pour qu'ils m'entendent : «Allez-y ! Allez-y ! Entrez! N'ayez pas peur! Vous êtes chez vous !». Voilà encore un endroit où je me suis si totalement revue, arrivant de la Guadeloupe où j'allais de temps à autre à la mairie emprunter des livres à une bibliothèque vieillotte et poussiéreuse, anémique aussi. Il y avait si peu de livres sur les rayonnages. Et puis, je suis arrivée à la mairie de Montreuil et dans la mairie, il y avait autrefois une très grande bibliothèque. Là, on avait encore le goût des livres reliés, de vert, autant que je m'en souvienne. Mais il y en avait tant. Alors, je suis entrée. Intimidée au début. Ne sachant par où commencer. Je ne sais pas pourquoi, j'ai commencé par le théâtre. Audiberti. Tout Audiberti. L'ordre alphabétique. J'ai suivi l'ordre alphabétique. Jusqu'à ce que je puisse trouver quelqu'un qui m'écoute, qui partage avec moi, échange, me guide aussi dans le dédale de cette histoire du théâtre.

Les personnalités dont on finit par observer le parcours dans le milieu artistique sont, la plupart du temps, des personnes qui ont été accompagnées, écoutées, entendues. Ce ne sont pas forcément des personnes faciles à vivre parce qu'elles sont dans l'urgence de dire, de 
faire, d'exprimer leur art. Ce ne sont pas des personnes soumises et lissées par les écoles dans lesquelles tous et toutes finissent par se ressembler. (Il faudrait aussi changer les écoles, la formation, bousculer la formation dans les écoles d'art).

Il convient d'encourager l'insolence, la pensée rebelle quand on la croise. Mais si, d'emblée, l'on craint que cette pensée rebelle ne se retourne contre vos prérogatives, alors, oui, on préférera peut-être un certain tokenisme, on privilégiera les visibles silencieux ayant l'échine souple. 
Que déserte la nuit

Et vienne une meute de criquets

Héler les lunes impavides.

Que déserte la nuit

Et bruisse une brassée de libellules

Au-dessus du fracas des échafauds qui tombent

Des tables sont dressées sur les mers en signe de bienvenue.

Débute l'épandage d'épices fortes

Par les champs d'habitudes.

Quand les hommes mis au ban prendront-ils part

Au grand accouplement

Des terres des mers du feu des volcans et du ciel au-dessus du silence ?

Que déserte la nuit

Et viennent déferler

des vagues aux déserts

À inonder les verbes immobiles

Et qu'explose le silence

En gerbes folles

Dans la surprise des yeux.

Un bateau s'éloigne de la terre.

Dernier écho des solitudes qu'il sème en son sillage

Hurle sa corne de brouillard 
Un bateau s'éloigne de la terre

Des femmes posent un tray

Sur leur fier équilibre

À l'odeur de sel durci des cordages

L'écho de la rudesse de leurs mains en rivières

Ô nostalgie des draps blancs sur la berge

Sur toutes les berges vivent femmes en attente

Sur toutes les berges feu sur l'attente

Brutale interruption des folles barbaries.

Sur toutes les rades

Feu sur l'attente

Feu à volonté

Que parlent les congas

Que les corps syncopés déshabillent la nuit

Que parlent les congas

Et mise à sac de l'ennui

De la peur

Et que les signes de connivence se dévoilent

Entre les hommes

Entre les femmes

Entre les désirs qui s'affirment à la portée des tours de guet. 
«DECOLONISER LES ARTS, C'EST VERITABLEMENT ALLER AU CEUUR DE QUESTIONS QUI SONT POSEES, NON PAS AUX DITS "DIVERS", MAIS A CEUX QUI SE VOIENT COMME UNE MAJORITE NATURELLE. DECOLONISER LES ARTS, C'EST SORTIR DE SON ENTRE-SOI. DECOLONISER LES ARTS, C'EST ACCEPTER DE SE DIRE QU'ON N'A PAS "L'ART INFUS" DE NOUS TOUS, QUELS QUE SOIENT NOS GENRES, NOS COULEURS, NOS ENTIERETES OU NOS PARCELLES. »

\section{GERTY DAMBURY}

